\title{
ORIGINAL
}

\section{VARIABILIDAD EN LA NOTIFICACIÓN DE REACCIONES ADVERSAS A LAS VACU- NAS DE LA GRIPE PANDÉMICA Y ESTACIONAL. TEMPORADAS 2009-2010 Y 2010-2011, COMUNITAT VALENCIANA}

\author{
Ana María Alguacil Ramos, José Antonio Lluch Rodrigo, Antonio Portero Alonso, Rosa Martín \\ Ivorra y Eliseo Pastor Villalba
}

Conselleria de Sanitat. Dirección General de Investigación y Salud Pública. Valencia

\begin{abstract}
RESUMEN
Fundamentos: La pérdida de confianza en la seguridad de las vacunas derivada de situaciones de alarma, como en el caso de la gripe pandémica, puede afectar tanto a las coberturas vacunales como a la sensibilidad frente a la notificación de las sospechas de reacciones adversas asociadas a vacunas (SRAAV). El objetivo del trabajo es describir los efectos adversos a la vacuna frente a la gripe pandémica notificados en la temporada 2009-2010 y comparar si existen diferencias con los descritos con la vacuna de la gripe estacional en las temporadas 2009-2010 y 2010-2011 en la Comunitat Valenciana (CV).

Métodos: Se realizó un estudio descriptivo de los individuos vacunados frente a la gripe que presentaron alguna SRAAV a la vacuna antigripal y que fue notificada a través del Sistema de Información Vacunal, durante las temporadas 2009-2010 (incluyendo la vacunación pandémica) y 2010-2011 en CV. se calcularon las tasas de notificación de sospechas de reacciones adversas asociadas a vacunas por cada mil dosis de vacunas administradas y sus intervalos de confianza al $95 \%$.

Resultados: Durante el periodo 2009-2010 la tasa de notificación de SRAAV para la vacuna de la gripe estacional fue de 0,02 por mil dosis administradas, para la vacuna pandémica de 0,95 . En el periodo 2010-2011 la tasa para la vacuna de la gripe estacional fue de 0,04 por mil.

Conclusiones: Durante las temporadas analizadas se incrementó el número de notificaciones de SRAAV para las vacunas pandémicas en comparación con el resto de vacunas antigripales. La mayor tasa de notificación de SRAAV correspondió al grupo de profesionales sociosanitarios, tanto para las vacunas frente a la gripe estacional como pandémica.

Palabras clave: Reacción adversa. Vacuna gripe. Profesionales sanita-
\end{abstract} rios. Sistema notificación reacciones a fármacos.

Correspondencia

Ana Maria Alguacil Ramos

Dirección General de Investigación y Salud Pública

Avd. Cataluña $n^{\circ} 21$

46020 Valencia

alguacil_ana@gva.es

\section{ABSTRACT \\ Variability in the Reporting of Adverse Reactions to the Pandemic and Seasonal Inluenza Vaccine. Seasons 2009-2010 and 2010-2011. Valencian Community, Spain.}

Background: The loss of confidence in the safety of vaccines derived from alarm conditions, as in the case of the flu pandemic may affect both vaccination coverage and the sensitivity to the reporting of suspected adverse reactions associated vaccines (SRAAV). The aim of the study is to describe the adverse effects reported to the vaccine against pandemic influenza in the 2009-2010 season and against seasonal influenza in 2009-2010 and 2010-2011 seasons, by type of vaccine, risk groups and by age group in the Valencian Community(CV).

Methods: A retrospective descriptive study has been made of the individuals presenting suspected adverse reactions to the influenza vaccine reported through the Vaccine Information System during the seasons 2009-2010 and 2010-2011 in the Valencian Community. 95\% confidence intervals were calculated.

Results: During the period 2009-2010 the reporting rate of suspected reactions for seasonal influenza vaccine was 0.020 per thousand doses administered, for the pandemic vaccine 0.95 . The reporting rate for seasonal influenza vaccine in the period 2010-2011 was 0.04 per thousand.

Conclusions: There was an increase in the number of reported suspected adverse reactions to the pandemic vaccines compared with the rest of influenza vaccines, during the seasons. The highest suspected adverse reaction reporting rate was for the group of health professionals partner for both vaccines against seasonal and pandemic influenza.

Key words: Adverse reaction. Influenza vaccines. Health care professionals. Adverse drug reaction reporting systems. 


\section{INTRODUCCIÓN}

En España se podría situar el inicio de la pandemia de gripe A cuando el Centro de Coordinación de Alertas y Emergencias (CCAES) del Ministerio de Sanidad, Política Social e Igualdad (MSPSI) recomendó reforzar los sistemas de vigilancia tras recibir la alerta sobre el brote el 24 de abril de 2009. El 26 de abril se notificaron en nuestro país los tres primeros casos posibles de infección por el nuevo virus de la gripe AH1N1, todos con antecedentes de viaje a México y que cumplían la definición de caso $^{1}$. Posteriormente se confirmó el diagnóstico de infección por el Centro Nacional de Microbiología perteneciente al Instituto de Salud Carlos III (ISCIII), centro de referencia a nivel nacional. El seguimiento y vigilancia del desarrollo del brote en España se llevó a cabo en el CCAES, junto con el Centro Nacional de Epidemiología (ISCIII) y de forma coordinada con las Comunidades Autónomas ${ }^{2,3}$.

El virus de la gripe A tiene la capacidad de variar genéticamente, determinando la aparición de nuevas cepas circulantes que generarán epidemias de intensidad y severidad variable si afectan a una gran población de individuos susceptibles. Este hecho fue lo que ocurrió durante la temporada 2009-2010 .

En la Región Europea fueron el Reino Unido y España los países que notificaron mayor número de $\operatorname{casos}^{5,6}$. En toda Europa se produjeron cerca de 3.000 muertes por la gripe pandémica.

La pandemia del año 2009 fue percibida por parte de la población como una enfermedad grave, lo que provocó que se viviera con "alarma". Tanto la situación de pandemia como la "rápida" comercialización de la vacuna frente a la gripe pandémica llevaron a la población y a muchos profesionales sanitarios a una percepción no sólo de alarma sino también de desconfian- za hacia las vacunas pandémicas disponibles. Las noticias difundidas por los diferentes medios de comunicación probablemente contribuyeron también a esta percepción ${ }^{7}$. Un factor que quizá contribuyó a que tanto la población como algunos profesionales sanitarios desconfiaran de la vacuna fue que tal vez no se informó de una forma clara y sencilla de que contenía adyuvantes ya conocidos y utilizados anteriormente ${ }^{8}$.

Tal como señalaron Apiñaniz $\mathrm{A}^{9}$ y Ayuso $\mathrm{E}^{10}$ en sus estudios, tanto la población como los profesionales sanitarios encuestados mostraron su rechazo a la vacunación frente a la gripe A por no disponer de información clara acerca de la misma y considerarla como poco ensayada. La consecuencia de este hecho se tradujo en que el porcentaje de población que se vacunó tanto en nuestra comunidad como en el resto de España fue bajo ${ }^{11}$. En la Comunitat Valenciana la mayor cobertura se produjo en el grupo de personas mayores de 60 años con patologías de riesgo, siendo del $30,62 \%{ }^{12}$.

En términos generales, las coberturas frente a la gripe en profesionales sanitarios vienen siendo en nuestro ámbito bastante bajas, encontrándose entre los motivos de no vacunación el temor a los efectos secundarios, tal y como señala un estudio realizado en el Hospital General Universitario de Alicante ${ }^{13}$, en el que el 18,8\% de las personas encuestadas dicen no vacunarse por este motivo. En otro estudio realizado en Grecia $^{14}$ se señala que la baja aceptación tanto para las vacunas antigripales estacionales y pandémicas por parte de los profesionales sanitarios era el temor a que fuesen poco seguras.

Las primeras vacunas antigripales comercializadas fueron aprobadas en EEUU en 1945 y dos años después se reconoció que los cambios antigénicos en la hemaglutinina de los virus gripales podía alterar la eficacia de la vacuna ${ }^{15}$. A lo largo 
de los años siguientes se han fabricado vacunas monovalentes, bivalentes y trivalentes. Desde 1978 la mayoría de vacunas han sido trivalentes y han incorporado virus gripales de los subtipos $\mathrm{A}(\mathrm{H} 1 \mathrm{~N} 1)$, A(H3N2) y el virus gripal B16.

Así mismo, durante décadas se han investigado diferentes adyuvantes para determinar la capacidad de aumentar la inmunogenicidad de las vacunas ${ }^{17-19}$. A lo largo de los años se han empleado vacunas antigripales adyuvadas con sales de aluminio, con MF59, vacunas antigripales virosomales $\mathrm{y}$, recientemente, se han introducido las adyuvadas con AS03, todas ellas con un demostrado amplio perfil de seguridad e inmunogenicidad ${ }^{20,21}$.

Las vacunas, al igual que el resto de medicamentos, se someten a ensayos clínicos previos a su comercialización. Durante las fases I, II, III se evalúa su seguridad, inmunogenicidad y eficacia ${ }^{22}$. En una última fase (IV), denominada postcomercialización y de duración mínima de 5 años tras la comercialización de la vacuna, se realiza un seguimiento más exhaustivo en cuanto a su seguridad en la población. Esta última fase permite la detección de posibles efectos adversos, de tasa de incidencia muy baja que no hayan acontecido en los ensayos clínicos previos ${ }^{23}$.

En el año 2009, ante la aparición de la pandemia de gripe A(H1N1)pdm09, la Food and Drug Administration (FDA) junto con la Agencia Europea del Medicamento (EMA) autorizaron la comercialización de vacunas con protección frente a esta cepa del virus de la influenza. Esta vacuna se elaboró mediante el procedimiento de vacunas molde, modelo o prototipo (mock-up vaccines) que introdujo la Comisión Europea y la Agencia Europea del Medicamento (EMA) en el año 2004, con el fin de utilizarse frente a la posible gripe aviar ${ }^{24}$. Esta estrategia consiste en el desarrollo de vacunas con cepas prototipo que puedan adaptarse rápi- damente en caso de aparición de un virus pandémico y así lograr una producción rápida de vacunas frente a la cepa epidémica adecuada $^{25}$.

Las vacunas elaboradas frente a la gripe A(H1N1)pdm09 se formularon por tanto con adyuvantes ampliamente utilizados, algunos ya conocidos, como el MF59 y otros mas nuevos, como el AS03, empleado en otras vacunas ${ }^{26}$.

Teniendo en cuenta los aspectos citados, es por lo que antes de comenzar la vacunación frente a la gripe pandémica en España y en la Comunitat Valenciana se planificó la monitorización de los posibles efectos adversos a las vacunas que se utilizaron para tal fin ${ }^{3}$.

En la Comunitat Valenciana se dispone de un sistema de registro de vacunas denominado Sistema de Información Vacunal (SIV). A través de él se recoge toda la información referente a actos vacunales de la población adscrita a la Comunitat Valenciana. A través del SIV se puede realizar la notificación de sospechas de reacciones adversas asociadas a vacunas on-line. Esta notificación se vincula a su vez al acto vacunal, quedando así reflejada en el historial vacunal del individuo ${ }^{27}$.

Mediante esta utilidad, el SIV permite la notificación al Centro Autonómico de Farmacovigilancia de la Comunitat Valenciana las sospechas de reacciones adversas asociadas a vacunas. El $98 \%$ de las declaraciones de eventos adversos asociados a la vacunación en el año 2008 se realizaron por este sistema ${ }^{28}$.

El objetivo del trabajo es describir los efectos adversos a la vacuna frente a la gripe pandémica notificados en la Comunitat Valenciana en la temporada 2009-2010 y frente a la gripe estacional en las temporadas 2009-2010 y 2010-2011, por tipo de vacuna, grupos de riesgo y por grupos de edad. 


\section{MATERIAL Y MÉTODOS}

Diseño del estudio: Se realizó un estudio descriptivo.

Fuente de información: Sistema de Información Vacunal de la Comunitat Valenciana: sospecha de reacción adversa a la vacuna antigripal en individuos vacunados frente a la gripe pandémica y estacional, por estar incluidos en alguno de los grupos de riesgo establecidos por el Sistema Nacional de Salud, durante las temporadas 2009-2010 y 2010-2011.

Periodos de estudio: del 1 de octubre de 2009 hasta el 28 de febrero de 2010 y del 27 de septiembre del 2010 hasta el 28 de febrero del 2011.

Criterios de inclusión en el estudio: Se incluyeron todas las sospechas de reacciones adversas notificadas.

Vacunas antigripales incluidas en el estudio.Temporada 2009-2010 y 20102011 (gripe estacional). Chiromas ${ }^{\circledR}$ de Laboratorio Novartis Vaccines and Diagnostics SL, vacuna virus fraccionados adyuvada con MF5929. Inflexal V $($ de laboratorio Crucell, vacuna de antígeno de superficie, inactivado, virosomal ${ }^{30}$. Influ$\operatorname{vac}{ }^{\circledR}$ del laboratorio Abbott Healthcare, vacuna antigripal inactivada de antígenos de superficie ${ }^{31}$.

Temporada 2009-2010 (gripe pandémica). Focetria ${ }^{\circledR}$ del laboratorio Novartis Vaccines and Diagnostics S.L, vacuna de antígenos de superficie, inactivada, adyuvada con MF59, en presentación multidosis conteniendo tiomersal y en presentación monodosis sin tiomersal ${ }^{32}$. Pandemrix ${ }^{\circledR}$ del laboratorio GlaxoSmithKline vacuna de virus fraccionados, inactivados, adyuvada con AS03, en presentación multidosis con tiomersal ${ }^{33}$. Panenza ${ }^{\circledR}$ del laboratorio Sanofi Pasteur MSD, vacuna de virus fraccionados, inactivados, sin adyu- vante, en presentación multidosis conteniendo tiomersal y en presentación monodosis sin tiomersal destinadas a la vacunación de las mujeres embarazadas ${ }^{34}$.

Variables: tipo de vacuna administrada, sexo, grupos de edad, tipo de reacción (local o general), grupos de riesgo, órganos y sistemas afectados y campaña antigripal.

Para la clasificación de las reacciones adversas notificadas se utilizó The Medical Dictionary for Regulatory Activities (MedDRA).

Una vez notificadas las reacciones adversas en el SIV, se validó y reclasificó la base de datos para su posterior análisis estadístico. Las tasas de notificación de sospechas de reacciones adversas asociadas a vacunas se calcularon por cada mil dosis de vacunas administradas.Se realizó el cálculo de los intervalos de confianza al 95\%. Para el análisis estadístico se utilizó el programa SPSS versión 14.0.

\section{RESULTADOS}

\section{Campaña de vacunación frente a la gripe estacional 2009-2010}

Se administraron 798.864 dosis. Hubo 16 $\left(0,020 \times 10^{3}\right)$ notificaciones de reacciones adversas a través del SIV de las cuales 6 $(37,5 \%)$ fueron en hombres y $10(62,5 \%)$ en mujeres. En la tabla 1se pueden ver las tasas por grupos de edad y grupos de riesgo.

Campaña de vacunación frente a la gripe pandémica 2009-2010

227.273 dosis de vacuna frente a la gripe pandémica y se registraron $216\left(0,953 / 10^{3}\right)$ notificaciones de reacciones adversas a través del SIV de las cuales $76(35,2 \%)$ fueron en hombres y $140(64,8 \%)$ en mujeres, 1 de ellas embarazada. En la tabla 1 se pueden ver las tasas por grupos de edad y grupos de riesgo. 
Tabla 1

Tasa de notificación de sospechas de reacciones adversas* por edad y grupo de riesgo

\begin{tabular}{|c|c|c|c|c|}
\hline \multicolumn{2}{|c|}{} & $\begin{array}{c}\text { Profesionales } \\
\text { sanitarios }\end{array}$ & $\begin{array}{c}\text { Población con } \\
\text { factores de riesgo } \\
>\text { 6 meses }\end{array}$ & Total \\
\hline $\begin{array}{c}\text { Campaña } \\
\text { antigripal }\end{array}$ & Edad & Tasa (IC95\%) & Tasa (IC95\%) & Tasa (IC95\%) \\
\hline \multirow{4}{*}{$\begin{array}{c}\text { Gripe } \\
\text { estacional } \\
2009 / 2010\end{array}$} & $<15$ & 0 & $0,034(-0,033-0,100)$ & $0,026(-0,025-0,077)$ \\
\cline { 2 - 5 } & $15-60$ & $0,152(-0,020-0,325)$ & $0,041(-0,001-0,081)$ & $0,037(0,012-0,063)$ \\
\cline { 2 - 5 } 798.864 dosis & $>60$ & 0 & $0,011(0,002-0,020)$ & $0,013(0,003-0,022)$ \\
\cline { 2 - 5 } & Total & $0,135(-0,018-0,288)$ & $0,016(0,001-0,026)$ & $0,020(0,010-0,030)$ \\
\hline \multirow{4}{*}{$\begin{array}{c}\text { Gripe } \\
\text { pandémica } \\
2009 / 2010 \\
227.273 \text { dosis }\end{array}$} & $<15$ & 0 & $2,712(1,743-3,681)$ & $2,700(1,735-3,665)$ \\
\cline { 2 - 5 } & $15-60$ & $8,801(7,058-10,545)$ & $1,052(0,766-1,337)$ & $2,257(1,898-2,615)$ \\
\cline { 2 - 5 } & $>60$ & $9,363(3,587-15,139)$ & $0,163(0,098-0,229)$ & $0,229(0,152-0,306)$ \\
\hline \multirow{2}{*}{$\begin{array}{c}\text { Gripe } \\
\text { estacional }\end{array}$} & Total & $8,851(7,181-10,521)$ & $0,366(0,284-0,449)$ & $0,953(0,826-1,080)$ \\
\cline { 2 - 5 } $2010 / 2011$ & $15-60$ & $0,582(0,179-0,986)$ & $0,025(-0,010-0,061)$ & $0,070(0,029-0,111)$ \\
\cline { 2 - 5 } 692.854 dosis & $>60$ & $1,563(0,032-3,094)$ & $0,018(0,006-0,030)$ & $0,026(0,012-0,040)$ \\
\cline { 2 - 5 } & Total & $0,736(0,320-1,153)$ & $0,023(0,011-0,036)$ & $0,040(0,025-0,054)$ \\
\hline
\end{tabular}

*Tasa por 1000 personas vacunadas

Campaña de vacunación frente a la gripe estacional 2010-2011. Se administraron 692.854 dosis de vacuna antigripal estacional y se registraron un total de 27 $\left(0,040 / 10^{3}\right)$ notificaciones de sospechas de reacciones adversas a través del SIV, 8 $(29,6 \%)$ en hombres y $19(70,4 \%)$ en mujeres.

El número de notificaciones para las vacunas frente a la gripe estacional en las campañas 2009-2010 y 2010-2011 (43) fue un $80 \%$ inferior al de las notificadas para las vacunas frente a la gripe pandémica (216).

Al analizar las notificaciones de reacciones por grupos de riesgo, el grupo de sujetos mayores de 6 meses con alguna patología de riesgo representó el $62,5 \%$ de las notificaciones para la vacuna frente a la gripe estacional en la temporada 2009-2010. El grupo de los profesionales socio sanitarios representó el 49,5\% de las notificaciones para la gripe pandémica $2009-2010$ y el $44,4 \%$ para la gripe estacional 2010-2011 (tabla 1).

En la figura 1 se presentan las tasas de notificaciones de reacciones adversas por tipo de vacuna, siendo la vacuna adyuvada con AS03 (Pandemrix ${ }^{\circledR}$ ) con una tasa de notificación de 2,517 por mil dosis administradas la que presentó la mayor tasa independientemente de la campaña.

En cuanto a la vacunación estacional, la vacuna virosomal presentó una tasa de notificación de 0,053 sospechas de reacciones adversas por mil dosis administradas, seguida de la fraccionada de subunidades con 0,025 y de la vacuna adyuvada con MF59 del 0,011. Durante la vacunación pandémica se registraron 158 notificaciones de sospechas de reacciones adversas a la vacuna adyuvada con AS03 (Pandemrix $\left.{ }^{\circledR}\right)$ y 56 notificaciones la vacuna adyuvada con MF59 (Focetria $\left.{ }^{\circledR}\right)$. 
Figura 1

Tasa de reacciones notificadas por tipo de vacuna

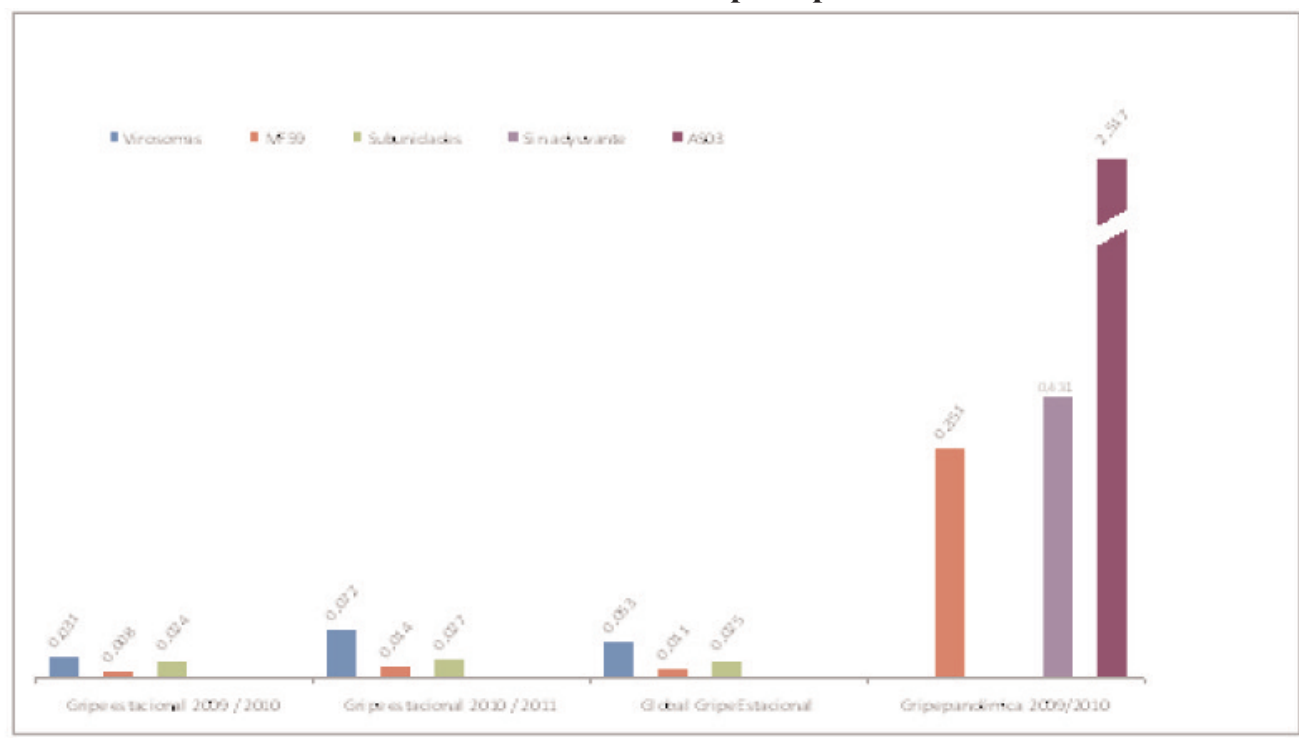

Figura 2

Trastornos generales y alteraciones en el lugar de administración

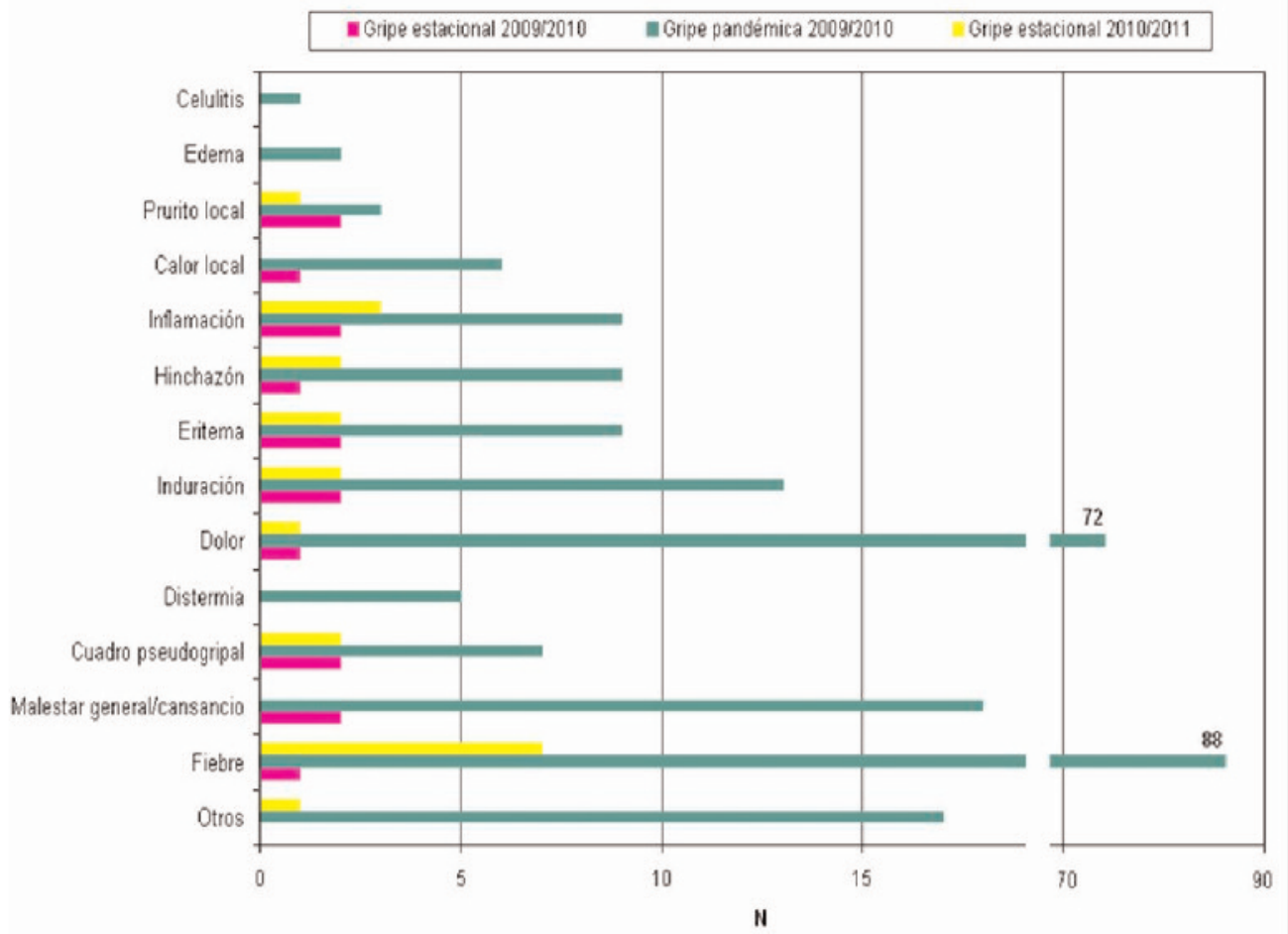




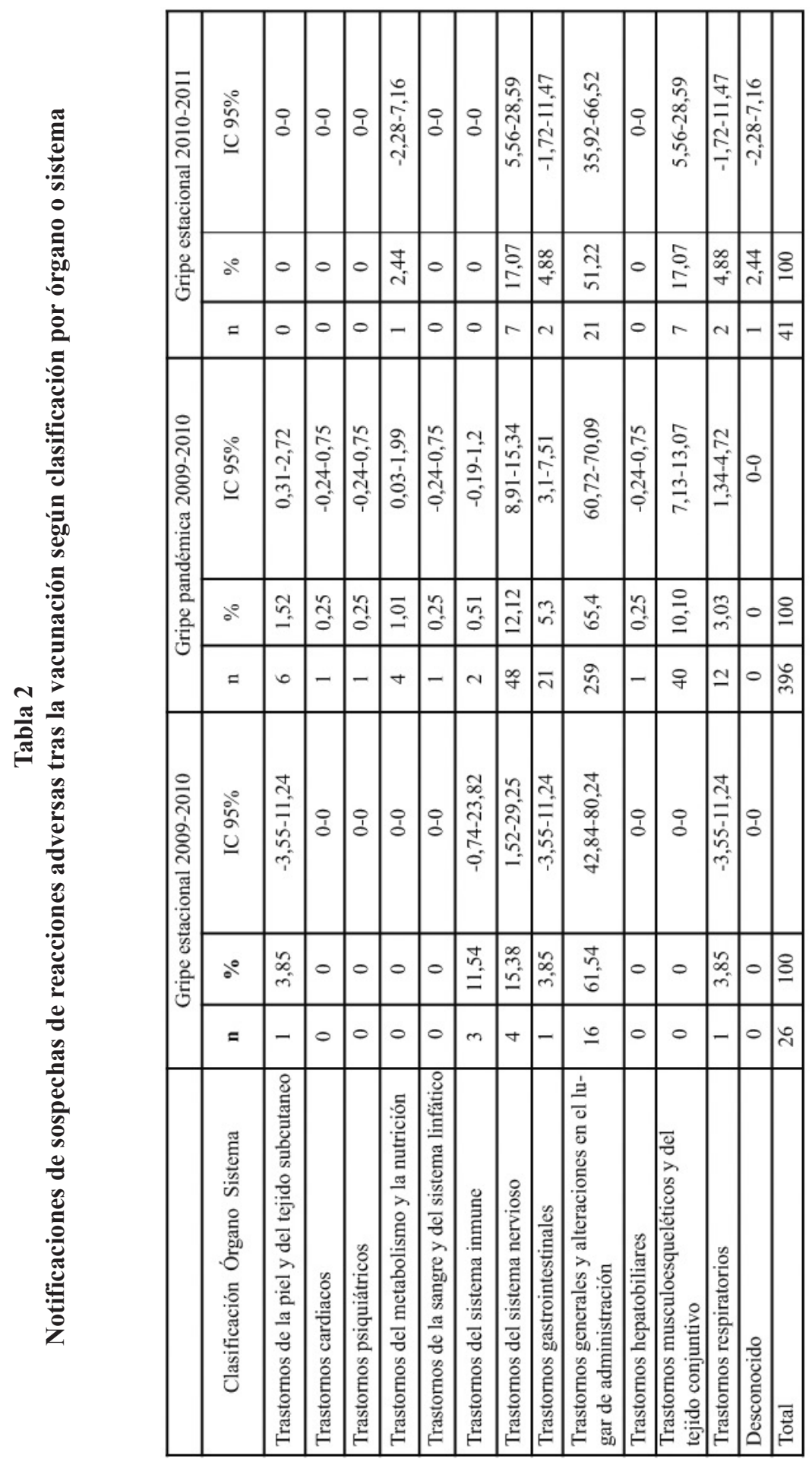


Respecto a las reacciones adversas producidas tras la vacunación por órganos y sistemas para cada uno de los periodos se muestran en la tabla 2.

Del total de reacciones notificadas, las correspondientes a trastornos generales y alteraciones en el lugar de administración representaron el $61,5 \%$ en la gripe estacional 2009-2010, el 65,4\% en la gripe pandémica y el 51,2\% en la gripe estacional 20102011. En la figura 2 se muestra el resto de la información.

\section{DISCUSIÓN}

Las tasas de notificación de sospechas de reacciones adversas asociadas a las vacunas antigripales durante el periodo de estudio fueron variables en las tres campañas. La tasa global de notificación para la vacuna pandémica fue 45 veces superior en relación con la vacuna frente a la gripe estacional de la campaña 2009-2010 y 24 veces superior en relación con la campaña 2010-2011. Mientras que la tasa de notificación global de la vacuna estacional 2010-2011 fue 2 veces superior a la de la gripe estacional 2009-2010. Todas estas diferencias fueron estadísticamente significativas.

Los CDC, mediante el US Vaccine Adverse Event Reporting System $(\text { VAERS })^{35}$, detectaron también una variabilidad en la notificación de las sospechas de reacciones adversas a las vacunas antigripales. No obstante, es necesario señalar que la principal diferencia con el sistema de declaración que se utiliza en la Comunitat Valenciana respecto al VAERS es que en el Sistema de Información Vacunal la notificación de las reacciones adversas sólo la realizan los profesionales sanitarios ${ }^{27}$.

Un estudio publicado recientemente por Sanchez Payá et $\mathrm{al}^{36}$, también señala una diferencia marcada en la notificación de sospechas de reacciones adversas entre las vacunas pandémica y estacional.
La tasa de notificación en nuestro estudio fue inferior a las obtenidas por Parreta E et $\mathrm{al}^{37}$ pero al igual que en nuestro estudio, según la clasificación MedDRA, el mayor porcentaje de reacciones notificadas fueron las correspondientes a trastornos generales y alteraciones en el lugar de administración $(55,9 \%)$, seguidas de reacciones del sistema nervioso $(33,2 \%)$ y de trastornos musculoesqueléticos $(20,3 \%)^{37}$.

Nuestros resultados coinciden también con el estudio publicado por Kraiguer A et $\mathrm{al}^{38}$, realizado en Eslovenia, acerca de la vigilancia de los efectos adversos notificados a las vacunas antigripales durante la gripe pandémica. Con la diferencia de que los trastornos gastrointestinales representaron un porcentaje ligeramente superior al encontrado en nuestro estudio.

En nuestro estudio, dentro de las reacciones enmarcadas en el grupo de trastornos generales y alteraciones en el lugar de administración, los mayores porcentajes se corresponden con la fiebre y el dolor en el punto de inoculación, coincidiendo con lo encontrado por Parreta E et $\mathrm{al}^{37}$ y Kraigher $\mathrm{A}^{38}$.

Durante el periodo de estudio no se notificó ningún caso de Guillain-Barre u otras alteraciones neurológicas graves.

En cuanto a la notificación de sospechas de reacciones adversas a vacunas por grupos de riesgo, para las tres campañas analizadas destacaron las tasas de notificación para el grupo de los profesionales sanitarios respecto a las notificadas en población general con factores de riesgo. En el estudio de Kraigher A. ${ }^{37}$ destaca que el $38 \%$ de las notificaciones analizadas correspondían al grupo de profesionales sanitarios.

El incremento de las notificaciones de sospecha de reacciones adversas en la vacunación frente al virus pandémico para todos los grupos de riesgo podría haberse debido a 
la especial vigilancia que se estableció por parte del Ministerio de Sanidad sobre las posibles reacciones adversas producidas tras la vacunación pandémica ${ }^{3}$ junto a una mayor sensibilización por parte de los profesionales sanitarios y la población, como consecuencia de la percepción que se tenía de que las vacunas eran poco seguras y se habían fabricado con premura, hechos a los que contribuyeron los diferentes medios de comunicación y las redes sociales ${ }^{39,40}$.

Está hipótesis se reforzaría por el descenso de notificaciones durante la vacunación estacional del año siguiente, cuando prácticamente desapareció de los medios de comunicación el debate sobre la seguridad de la vacuna antigripal. Cabe señalar que para la vacunación estacional 2010-2011 la tasa de notificación obtenida fue superior a la estacional 2009-2010, probablemente consecuencia de la vacunación pandémica del año anterior, si bien estas diferencias no fueron estadísticamente significativas. Todo ello también explicaría la mayor tasa de notificación de sospechas de reacciones adversas a las vacunas antigripales pandémicas adyuvadas con AS03 y MF59, pudiéndose deber, por tanto, a una mayor sensibilidad en la notificación más que a la propia reactogenicidad de las vacunas.

Por otra parte, el mayor número de notificaciones de sospecha de reacciones adversas en el grupo de profesionales sociosanitario podría ser consecuencia de la mayor accesibilidad al sistema de declaración junto con una percepción de que las vacunas no estaban lo suficientemente ensayadas ${ }^{10}$.

Por último, señalar que tanto las vacunas que se adquirieron para la vacunación frente a la gripe estacional como frente a la gripe pandémica son vacunas seguras y que las sospechas de reacciones adversas que se notificaron en la Comunitat Valenciana coinciden con las descritas en sus fichas técnicas $^{29-34}$
El estudio realizado, a diferencia del resto de estudios realizados en España sobre reacciones adversas a vacunas antigripales, analiza las sospechas de reacciones adversas notificadas directamente por el profesional sanitario en un sistema de registro nominal de vacunas, como es el SIV, sin emplear en ningún caso la encuesta presencial o telefónica. Esto permite, entre otras ventajas, no sólo la detección y análisis de reacciones adversas precoces sino también las ocurridas tras un periodo de tiempo más prolongado. De acuerdo con los datos facilitados por el Centro Autonómico de Farmacovigilancia de la Comunitat Valenciana el 91,14\% de las notificaciones de sospechas de reacciones adversas a la vacuna pandémica se registraron a través del SIV, por lo que se puede afirmar que este sistema mantuvo la exhaustividad en el registro en la situación especial que supuso la vacunación pandémica.

La principal limitación de nuestro estudio se basa en que nos encontramos ante una notificación pasiva por parte del profesional sanitario, lo cual puede derivar en algunos casos en una infranotificación, tal como se recoge en otros trabajos ${ }^{41,42}$.

\section{AGRADECIMIENTOS}

Al Servicio de Ordenación y Control del Medicamento de la Comunitat Valenciana y en especial al Centro Autonómico de Farmacovigilancia.

\section{BIBLIOGRAFÍA}

1.Ministerio de Sanidad y Política Social. Subcomité de vigilancia. Plan Nacional de preparación y respuesta ante una pandemia de gripe. [Citado el 09.09.2009] Disponible en:

http://www.msc.es/profesionales/saludPublica/gripeA/estrategiaVigilancia.htm

2.European Medicines Agency. CHMP Recommendations for the Pharmacovigilance Plan as part of the Risk Management Plan to be submitted with the Marketing Authorisation Application for a Pandemic Influenza Vaccine. London. 2009. Disponible en : http://www.ema.europa.eu 
3.Agencia Española de Medicamentos y Productos Sanitarios. Plan de farmacovigilancia de las vacunas pandémicas. Oct. 2009. Disponible en: http://www.aemps.es/profHumana/farmacovigilancia/planVacunasPandemicas_gr ipeA_H1N1.htm

4.Glezen WP. Serious morbidity and mortality associated with infuenza epidemics. Epidemiol Rev. 1982;4:25-44.

5.European Centre for Disease Prevention and Control (ECDC). European Influenza Surveillance Network. Disponible en: http://www.ecdc.europa.eu/en/activities/surveillance/EISN/Pages/index.aspx

6.Red Nacional de Vigilancia Epidemiológica. Sistema de Vigilancia de la gripe en España. Disponible en:.http://vgripe.isciii.es/gripe/inicio.do

7.Sanchez Martos J. La información sobre la salud en los medios de Comunicación. Rev Esp Com Sal. 2010;1(2): 68-76.

8.Vírseda S, Restrepo MA, Arranz E, Magán-Tapia P, Fernandez-Ruiz M, Gómez de la Camara A, et al. Seasonal and Pandemic A (H1N1) 2009 influenza vaccination coverage and attitudes among health-care workers in a Spanish University Hospital. Vaccine. $2010 \mathrm{Jul}$ 5;28(30):4751-7.

9.Apiñaniz A, López-Picado A, Miranda-Serrano E, Latorre A, Cobos R, Parraza-Diez N, et al. Estudio transversal basado en la población sobre la aceptabilidad de la vacuna y la percepción de la gravedad de la gripe A/H1N1: opinión de la población general y de los profesionales sanitarios. Gac Sanit. 2010;24(4):314-320.

10.Ayuso C, Pérez J, Martínez I, Escobar E.. Campaña vacunal frente a Gripe A. Opiniones sobre la inmunización entre el Colectivo Médico. Rev Clín Med Fam. 2010; 3 (2): $88-92$

11.Ministerio de Sanidad, Política Social e Igualdad. Coberturas de Vacunación. [Citado el 05.05.2011] Disponible en:

http://www.msps.es/profesionales/saludPublica/prevPromocion/vacunaciones/coberturas.htm\#decimo

12.Pastor E, Portero A, Martin R, Alguacil AM, Lopez A ,Miralles MT et al. Prevención y vigilancia de la gripe en la Comunitat Valenciana. Temporada 2009-2010. Conselleria de Sanitat 2010. Informe de Salud n ${ }^{\circ} 121$. Disponible en:

http://www.sp.san.gva.es/biblioteca/publicacion_dgsp.js p?cod_pub_ran $=56709 \& \operatorname{tacc}=17$

13.Galicia-García MD, Gonzalez-Torga A, Garcia-Gonzalez C, et al. Vacunación de gripe en trabajadores sanitarios. Por qué se vacunan y por qué no se vacunan. Enfer. Infecc. Microbiol Clin. 2006; 24(7):413-7.
14. Rachiotis G, Mouchtouri VA, Kremastinou J, Gourgoulianis K, Hadjichristodoulou C. Low acceptance of vaccination against the 2009 pandemic influenza $\mathrm{A}(\mathrm{H} 1 \mathrm{~N} 1)$ among healthcare workers in Greece. Euro Surveill. 2010; 15(6): pii=19486. Disponible en: www.eurosurveillance.org/ViewArticle.aspx?ArticleId $=19486$

15.Francis T. A new type of virus from epidemic influenza. Science. 92:405-8.

16. World Health Organization Recommendations for influenza vaccines. Disponible en: http:/www.who.int/csr/disease/influenza/vaccinerecommendations/en/index.html

17.Meyer HM Jr, Hopps HE, Parkman PD, Ennis FA. Review of exiting vaccines for influenza. Am J Clin Pathol. 1978; 70 (1 suppl): 146-152.

18. Vogel FR. Improving vaccine performance with adjuvants. Clin Infect Dis. 2000; 30 Suppl 3: S266270 .

19.Petrovsky N, Aguilar JC. Vaccine adjuvants: current state and future trenes. Inmunol Cell Biol. 2004;82:488-96.

20.Franco D A, Giraldo M; Patiño PJ. Papel de los adyuvantes en la modulación de la respuesta inmune. Rev Col Cienc Pec. 2004; Vol 17:3.

21. O’Hagan D. MF59 es un adyuvante para vacunas seguro y potente que potencia la protección frente a la infección por el virus de la gripe. Expert Rev Vaccines. 2007;6: 699-710.

22. López A, Montané E. Aspectos prácticos en la evaluación de ensayos clínicos con vacunas profilácticas frente a enfermedades infecciosas. Med Clin (Barc). 2010;135(15):707-712.

23. Peiró S. La seguridad de los medicamentos: autorización y vigilancia post-comercialización. Humanitas, Humanidades Médicas. 2005 ; 8 : 87-99

24. European Medicines Agency. Authorisation procedures. [Citado el 10.11.2011] Disponible en http://www.ema.europa.eu/ema/index.jsp?curl=pages/ special_topics/q_and_a/q_and_a_detail_000080.jsp\&j senabled $=$ true

25. Roman F, Vaman T, Gerlach B et al Immunogenicity and safety in adults of one dose of influenza A H1N1v 2009 vaccine formulated with and without AS03A-adjuvant: Preliminary report of an observerblind, randomised trial. Vaccine. 2010; 1740-45. 
26. Committee for Proprietary Medicinal Products (CPMP). Guideline on submission of marketing authorization applications for pandemic influenza vaccines through the centralized procedure. Londres: European Agency for the Evaluation of Medicinal Products; 2004.

27. Pastor E, Martín R, Alguacil AM, Portero A, Lluch JA, et al. Sistema de Información Vacunal. $2^{\circ}$ Edición.Generalitat. Conselleria de Sanitat. 2009. Disponible en: http://www.sp.san.gva.es/biblioteca/publicacion_dgsp.jsp?cod_pub_ran=19281\&tacc $=15$

28.Alguacil AM; Gomar MJ, Portero A, Pastor E, Ivorra R, Lluch J. Farmacovigilancia activa y vacunas: el papel de las t.i.c. en la declaración de las reacciones adversas en la Comunitat Valenciana. Comunicación presentada en IX Jornadas de Farmacovigilancia Oviedo. Junio 2009 . Disponible en : http://www.aemps.gob.es/eventosCongresos/2009/doc s/IX-Jornadas-FV_junio-2009/libro-Resumen_IX-Jornadas-FV_junio-2009.pdf

29. Ficha técnica de Chiromas ${ }^{\circledR}$. [Citado el 10.11.2011] Disponible en: http://www.aemps.gob.es/cima/especialidad.do?meto$\mathrm{do}=$ verFichaWordPdf $\&$ codigo $=63566 \&$ formato $=$ pdf $\&$ formulario=FICHAS \&file=ficha.pdf

30.Ficha técnica de Inflexal V®. [Citado el $10.11 .2011] \quad$ Disponible en: http://www.aemps.gob.es/cima/especialidad.do?meto$\mathrm{do}=$ verFichaWordPdf $\&$ codigo $=64459 \&$ formato $=$ pdf $\&$ formulario=FICHAS\&file=ficha.pdf

31. Ficha técnica de Influvac ${ }^{\circledR}$.[Citado el 10.11.2011] Disponible en: http://www.aemps.gob.es/cima/especialidad.do metodo $=$ verFichaWordPdf\&codi go $=57523 \&$ formato $=$ pdf $\&$ formulario $=$ FICHAS $\&$ file $=$ ficha.pdf

32.Ficha técnica de Focetria ${ }^{\circledR}$. [Citado el 10.11.2011] Disponible en:

http://www.ema.europa.eu/ema/index.jsp?curl=pages/ medicines/human/medicines/000710/human med 00 0796.jsp\&mid=WC0b01ac058001d124

33.Ficha técnica de Pandemrix ${ }^{\circledR}$.[Citado el 10.11.2011] Disponible en:

http:/www.ema.europa.eu/ema/index.jsp?curl=pages/ medicines/human/medicines/000832/human_med_00 0965.jsp\&mid=WC0b01ac058001d124

34.Ficha técnica de Panenza®.[Citado el 10.11.2011] Disponible en: http://www.aemps.gob.es/cima/especialidad.do metodo $=$ verFichaWordPdf\&codi $\mathrm{go}=71627 \&$ formato $=$ pdf $\&$ formulario $=$ FICHAS $\&$ file $=$ ficha.pdf
35. Centers for Disease Control and Prevention (CDC). Safety of influenza a(h1n1) 2009 monovalent vaccines-United States, October 1-November 24, 2009. MMWR. 2009;58:1351-6.

36. Sánchez-Payá J, Hernández-Garcia I, Barrenengoa-Sañudo J, Camargo-Angeles R, Rincon A, Romeiro-Candeira S.. Frecuencia de reacciones adversas y factores asociados tras la administración de la vacuna de la gripe en personal sanitario durante la temporada 2009-2010. Rev Esp Salud Pública. 2010;84:851-9.

37. Parreta E, Ianniello B, Ferrazin F, Rossi F, Capuano A. Italian post-marketing surveillance for adverse event reports alter MF59-adjuvanted vaccination. Vaccine. 2011;29: 3708-13.

38. Kraigher A, Vcakar V. Surveillance of adverse events following immunization against pandemic influenza in Slovenia in season 2009/10. Vaccine. 2010; 28 : 5467-72.

39.Bish A, Yardley L, Nicoll A, Michie S. Factors associated with uptake of vaccination against pandemic influenza: A systematic review. Vaccine. 2011; 29: $6472-84$

40.Black S, Eskola J, Siegrist CA., Hasley N, MacDonal N, Law B et al. Importance of background rates of disease in assessment of vaccine safety during mass immunisation with pandemic H1N1 influenza vaccines. Lancet. 2009; 374: 2115-22.

41.Schumacher Z, Bourquin C, Heininger U. Surveillance for adverse events following immunization (AEFI) in Switzerland,1991-2001. Vaccine. 2010; 28: 4059-4064

42.Liang XF, Li L, Liu DW, Li KL, Wu WD, Zhu BP, et al. Safety of Influenza A (H1N1) Vaccine in Postmarketing Surveillance in China. N Engl J Med. 2011;364(7):638-47. 